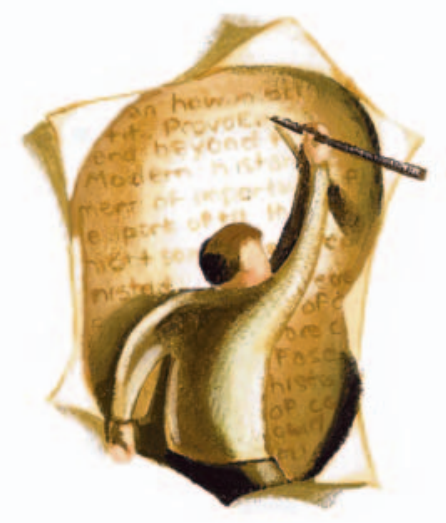

Analyzing the risks

\section{of cesarean delivery}

Shiliang Liu and colleagues conclude that the risks of severe maternal morbidity associated with planned cesarean delivery are higher than those associated with planned vaginal delivery. ${ }^{1}$ However, a cross-sectional study of associations is nondirectional and one cannot infer either the presence of causality or its direction.

During the I4-year study period, the rate of cesarean deliveries was increasing and morbidity was probably decreasing. Associations between 2 heterogeneous data sets are not meaningful. One needs data from a recent homogeneous time period to produce statistical associations that are relevant for current practice.

Finally, the authors acknowledge that although the morbidity rate differences were statistically significant, the absolute differences were small. The authors do not state which, if any, of these small differences were clinically significant.

\section{Vincent V. Richman PhD \\ Professor of Accounting \\ School of Business and Economics \\ Sonoma State University \\ Rohnert Park, Calif.}

Competing interests: None declared.

\section{REFERENCE}

I. Liu S, Liston RM, Joseph KS, et al, for the Maternal Health Study Group of the Canadian Perinatal Surveillance System. Maternal mortality and severe morbidity associated with low-risk planned cesarean delivery versus planned vaginal delivery at term. CMAJ 2007;176:455-60.

DOI:I0.1503/cmaj.107005I

\section{Treatment of septic}

\section{arthritis}

Raheem Kherani and Kam Shojania recently provided a comprehensive overview of septic arthritis; however, I have some concerns about the antimicrobial therapies outlined in their article. ${ }^{1}$ The authors recommend that grampositive cocci identified in Gram's staining of synovial fluid should be treated with cefazolin if the infection was acquired in the community. This treatment recommendation does not consider the emergence of community-acquired methicillin-resistant Staphylococcus aureus in Canada. ${ }^{2}$ When one is treating an infection that leads to joint destruction or sepsis, it is prudent to include vancomycin as a first-line agent until the antibiotic susceptibility of the organism is known. ${ }^{3}$

My second concern is the authors' recommendation that cefazolin and gentamicin should be used to treat cases in which Gram's staining does not show bacteria. Most treatment recommendations for this scenario include a third-generation cephalosporin and vancomycin for empiric coverage of the most common bacterial pathogens. Neisseria gonorrhoeae would also be covered by this broader regimen; it is a common cause of septic arthritis in patients at risk for sexually transmitted infections. With the rising incidence of gonorrhea in Canada ${ }^{4}$ and the frequency of a negative result of Gram's staining with gonococcal arthritis, this organism should at least be considered when treating septic arthritis.

\section{Cheryl Main MD BSc}

Medical Microbiologist

Hamilton Health Sciences

Hamilton, Ont.

Competing interests: None declared.

\section{REFERENCES}

I. Kherani RB, Shojania K. Septic arthritis in patients with pre-existing inflammatory arthritis. CMAJ 2007;176:1605-8

2. Hawkes M, Barton M, Conly J, et al. Communityacquired MRSA: superbug at our doorstep. CMAJ 2007;176:54-6.

3. Barton-Forbes M, Hawkes M, Moore D, et al.
Guidelines for the prevention and management of community associated methicillin resistant Staphylococcus aureus (CA-MRSA): a perspective for Canadian health care practitioners. Can J Infect Dis Med Microbiol 2006; I7(Suppl C):IB-24B .

4. Mann J, Kropp R, Wong T, et al. Gonorrhea treatment guidelines in Canada: a 2004 update. CMAJ 2004;I7I:I345-6.

DOI:Io.I503/cmaj.I070080

\section{[The authors respond:]}

We thank Cheryl Main for her comments on the antimicrobial coverage for both gram-positive and gram-negative septic arthritis that we recommended in our article. ${ }^{1}$ Our review focused on nongonococcal septic arthritis in patients with pre-existing inflammatory arthritis, and space constraints meant that we could not elaborate on many special circumstances.

Unfortunately, the literature on community-acquired gram-positive septic arthritis in this population is limited. One case series of 59 patients with septic arthritis (in which 15 of the cases were due to MRSA and 44 were not) includes several patients with pre-existing rheumatic disease but does not provide details on the nature of their rheumatic disease. $^{2}$ The authors of this case series suggest considering empiric treatment for MRSA infection in patients with septic arthritis if there are risk factors such as recent admission to hospital, known infection or colonization with MRSA, multiple comorbidities in addition to the rheumatic disease, injection drug use or residence in communities known to have a high prevalence of communityacquired MRSA infections. These suggestions are consistent with our interpretation of the guidelines referenced by Main. ${ }^{3,4}$ Hawkes suggests that people of First Nations or African-American heritage, athletes who participate in contact sports, injection drug users, men who have sex with men, military personnel, inmates of correctional facilities, veterinarians, pet owners and pig farmers may be at increased risk of developing MRSA infections. ${ }^{3}$ As a result, our recommendations continue to be to use cefazolin empirically. For patients known to have risk factors for MRSA infections, vancomycin should be included in the 